\title{
CURVATURE AND THE EIGENFORMS OF THE LAPLACE OPERATOR
}

\author{
V. K. PATODI
}

\section{Introduction}

Let $X$ be a $d$-dimensional compact oriented Riemannian manifold of class $C^{\infty}$ without boundary, $\wedge^{p}$ the space of smooth exterior $p$-forms, $d: \wedge^{p} \rightarrow \wedge^{p+1}$ the operator of exterior differentiation, $d^{*}: \wedge^{p+1} \rightarrow \wedge^{p}$ the adjoint of $d$ with respect to the Riemannian metric, and $\Delta=-\left(d d^{*}+d^{*} d\right)$ the Laplace operator acting on exterior $p$-forms for $0 \leq p \leq d$. It is known that $\Delta: \wedge^{p} \rightarrow \wedge^{p}$ has an infinite sequence

$$
0 \geq \lambda_{1} \geq \lambda_{2} \geq \cdots \geq \lambda_{n} \cdots \downarrow-\infty
$$

of eigenvalues, each of which is repeated as many times as its multiplicity indicates, and the corresponding sequence $\left\{\varphi_{n}\right\}$ of eigenforms forms a complete orthonormal set in the space $\wedge^{p}$ with Riemannian inner product. The sum

$$
\sum_{n \geq 0} \exp \left(\lambda_{n} t\right) \varphi_{n}(x) \otimes \varphi_{n}(y)
$$

converges uniformly on compact subsets of $(0, \infty) \times X$ to the fundamental solution $e^{p}(t, x, y)$ of the operator $\partial / \partial t-\Delta$ acting on $p$-forms, and the trace $Z^{p}=\sum_{n \geq 0} \exp \left(\lambda_{n} t\right)$ can be expressed as the integral over the manifold of the pole $\operatorname{Tr} e^{p}=\sum_{n \geq 0} \exp \left(\lambda_{n} t\right)\left\langle\varphi_{n}, \varphi_{n}\right\rangle,\left\langle\varphi_{n}, \varphi_{n}\right\rangle$ being the Riemannian inner product of $p$-forms at a point of $X$, that is,

$$
Z^{p}=\int_{X} \operatorname{Tr} e^{p} .
$$

Let $Z$ be the alternating sum of $Z^{p}$, that is,

$$
Z=\sum_{p=0}^{d}(-1)^{p} Z^{p}
$$

and $\operatorname{Tr} e=\sum_{p=0}^{d}(-1)^{p} \operatorname{Tr} e^{p}$. It is proved in [2] that

Communicated by I. M. Singer, April 20, 1970. 


$$
Z=\int \operatorname{Tr} e=\text { the Euler Poincaré characteristic of } X .
$$

On the other hand Chern's extension of the classical Gauss-Bonnet formula states that for manifolds of even dimension $d$, the Euler Poincare characteristic $E=\int C$, where $C$ is a homogeneous polynomial of degree $d / 2$ in the components of the curvature tensor. On account of these two results, one can expect that some fantastic cancellation will also take place in the alternating pole sum $\operatorname{Tr} e$ and one will have the following theorem (see [2]):

Theorem. Let $e^{p}(t, x, y)$ be the fundamental solution of the heat operator $\partial / \partial t-\Delta$ acting on $p$-forms, $0 \leq p \leq d$, and let

$$
\operatorname{Tr} e=\sum_{p=0}^{d}(-1)^{p} \operatorname{Tr} e^{p} .
$$

Then

$$
\operatorname{Tr} e=\left\{\begin{array} { l } 
{ 0 } \\
{ C }
\end{array} + 0 ( t ) \text { for } d \left\{\begin{array}{l}
\text { odd } \\
\text { even }
\end{array}, \quad t \downarrow 0 .\right.\right.
$$

The result with $O(t)=0$ is a triviality for odd $d$ in view of the Poincare duality $\operatorname{Tr} e^{p}=\operatorname{Tr} e^{d-p}$. The authors [2] have proved result (1) for $d=2$. The main purpose of this paper is to prove the above theorem for all even $d$. $\S 2$ and $\S 3$ are devoted to some preliminaries, and in $\S 4$ the fundamental solution for the heat operator $\partial / \partial t-\Delta$ is constructed. Finally in $\S 5$ we prove the theorem.

The author wishes to express his thanks to Professors M. S. Narasimhan and S. Ramanan for their suggestions which enabled him to simplify the proof.

\section{Algebraic preliminaries}

Let $V$ be a finite dimensional vector space over the field $R$ of real numbers, $d$ its dimension, $V^{*}$ the dual space of $V$, and $A$ a linear operator from $V$ into itself. Then for $1 \leq p \leq d$, there are two naturally defined linear operators $\wedge^{p} A$ ( $p$-th exterior power of $A$ ) and $D^{p} A$ ("derivation extension" of $A$ ) from $\wedge^{p} V$ into itself,

$$
\begin{array}{r}
\left(\wedge^{p} A\right)\left(v_{1} \wedge \cdots \wedge v_{p}\right)=\left(A v_{1}\right) \wedge \cdots \wedge\left(A v_{p}\right) \\
\left(D^{p} A\right)\left(v_{1} \wedge \cdots \wedge v_{p}\right)=\sum_{r=1}^{p} v_{1} \wedge \cdots \wedge v_{r-1} \wedge\left(A v_{r}\right) \wedge v_{r+1} \wedge \cdots \wedge v_{p} \\
v_{1}, \cdots, v_{p} \in V
\end{array}
$$

We define $\wedge^{0} A, D^{0} A$ respectively to be the identity endomorphism, zero 
endomorphism of the field of scalars. For a linear operator $B$ of $V$ into itself, we shall denote the trace of $B$ by $\operatorname{Tr} B$.

Lemma 2.1. Let $A_{1}, \cdots, A_{k}$ be linear operators from $V$ into itself, $k \leq d$. Then

$$
\begin{aligned}
\sum_{p=0}^{d}(-1)^{p} \operatorname{Tr}\left(D^{p} A_{1} \circ \cdots \circ D^{p} A_{k}\right) & \text { if } k<d, \\
= & \left\{\begin{array}{c}
0 \\
(-1)^{d} \text { coefficient of } x_{1} \cdots x_{k} \text { in } \operatorname{det}\left(x_{1} A_{1}\right. \\
\left.+x_{2} A_{2}+\cdots+x_{k} A_{k}\right)
\end{array} \text { if } k=d .\right.
\end{aligned}
$$

Proof. Let $x_{1}, \cdots, x_{k}$ be $k$-parameters. Then we have

$$
\begin{aligned}
\operatorname{det}\left(I-e^{x_{1} A_{1}} \cdots e^{x_{k} A_{k}}\right) & =\sum_{p=0}^{d}(-1)^{p} \operatorname{Tr}\left(\wedge^{p}\left(e^{x_{1} A_{1}} \cdots e^{x_{k} A_{k}}\right)\right) \\
& =\sum_{p=0}^{d}(-1)^{p} \operatorname{Tr}\left(e^{x_{1} D^{p_{A}}} \cdots e^{x_{k} D^{p_{A}} A_{k}}\right),
\end{aligned}
$$

and the lemma follows by equating the coefficients of $x_{1} \cdots x_{k}$ in $\operatorname{det}\left(I-e^{x_{1} A_{1}}\right.$ $\left.\cdots e^{x_{k} A_{k}}\right)$ and $\sum_{p=0}^{d}(-1)^{p} \operatorname{Tr}\left(e^{x_{1} D^{p} A_{1}} \ldots e^{x_{k} D^{p_{A}}}\right)$.

For any integer $p$ between 0 and $d$, the map $\varphi_{p}$ from $\left(V^{*} \otimes V\right) \times\left(V^{*} \otimes V\right)$ into Hom $\left(\wedge^{p} V, \wedge^{p} V\right)$, defined by

$$
\varphi_{p}(A, B)=\left(D^{p} A\right) \circ\left(D^{p} B\right), \quad A, B \in V^{*} \otimes V \approx \operatorname{Hom}(V, V),
$$

is bilinear and therefore defines a linear map $\tilde{\varphi}_{p}$ from $V^{*} \otimes V \otimes V^{*} \otimes V$ into Hom $\left(\wedge^{p} V, \wedge^{p} V\right)$. We shall denote the image of an element $A \in V^{*} \otimes V \otimes V^{*} \otimes V$ under $\tilde{\varphi}_{p}$ by $D^{p} A$.

Lemma 2.2. Let $l_{1}, l_{2}$ be nonnegative integers such that $l_{1}+2 l_{2}<d, \sigma$ be a permutation of $\left\{1, \cdots, l_{1}+l_{2}\right\}, A_{\sigma(1)}, \cdots, A_{o\left(l_{1}\right)}$ be arbitrary elements of $V^{*} \otimes V$, and $A_{\sigma\left(l_{1}+1\right)}, \cdots, A_{\sigma\left(l_{1}+l_{2}\right)}$ be arbitrary elements of $V^{*} \otimes V \otimes V^{*} \otimes V$. Then

$$
\sum_{p=0}^{d}(-1)^{p} \operatorname{Tr}\left(D^{p} A_{1} \circ \cdots \circ D^{p} A_{l_{1}+l_{2}}\right)=0 .
$$

Proof. Since every element of $V^{*} \otimes V \otimes V^{*} \otimes V$ can be written as a finite sum $\sum \lambda_{i} \otimes \mu_{i}, \lambda_{i}, \mu_{i} \in V^{*} \otimes V$, Lemma 2.2 immediately follows from Lemma 2.1 .

Lemma 2.3. Suppose that the dimension $d$ of the vector space $V$ is an even integer $2 n$. Let $A$ be an arbitrary element of $V^{*} \otimes V \otimes V^{*} \otimes V, e_{1}, \cdots, e_{2 n}$ be a basis for $V$, and $e_{1}^{*}, \ldots, e_{2 n}^{*}$ be the dual basis for $V^{*}$. Suppose that $A=$ $\sum a_{i j k l} e_{i}^{*} \otimes e_{j} \otimes e_{k}^{*} \otimes e_{l}$. Then 


$$
\sum_{p=0}^{a}(-1)^{p} \operatorname{Tr}\left(\left(D^{p} A\right)^{n}\right)=\sum \varepsilon_{\sigma} \varepsilon_{\delta} a_{\sigma(1) \delta(1) \sigma(2) \delta(2)} \cdots a_{\sigma(2 n-1) \delta(2 n-1) \sigma(2 n) \delta(2 n)},
$$

where on the right hand side the sum runs over all permutations $\sigma, \delta$ of $\{1, \cdots, 2 n\}$ and $\varepsilon_{\sigma}, \varepsilon_{\delta}$ are the signatures of the permutations $\sigma, \delta$ respectively.

Proof. We have

$$
\begin{aligned}
& \sum_{p=0}^{d}(-1)^{p} \operatorname{Tr}\left(\left(D^{p} A\right)^{n}\right) \\
& =\sum_{p=0}^{d}(-1)^{p} \operatorname{Tr}\left(\sum a_{i_{1} j_{1} i_{2} j_{2}} \cdots a_{i_{2 n-1} j_{2 n-1} i_{2 n} j_{2 n}} D^{p}\left(e_{i_{1}}^{*} \otimes e_{j_{1}}\right)\right. \\
& \left.\quad \circ D^{p}\left(e_{i_{2}}^{*} \otimes e_{j_{2}}\right) \circ \cdots \circ D^{p}\left(e_{i_{2 n-1}}^{*} \otimes e_{j_{2 n-1}}\right) \circ D^{p}\left(e_{i_{2 n}}^{*} \otimes e_{j_{2 n}}\right)\right) \\
& =\sum_{\substack{i_{1}, \cdots, i_{2 n} \\
j_{1}, \cdots, j_{2 n}}} a_{i_{1} j_{1} i_{2} j_{2}} \cdots a_{i_{2 n-1} j_{2 n-1} i_{2 n} j_{2 n}} \times \text { coefficient of } x_{1} \cdots x_{2 n} \text { in } \\
& \quad \operatorname{det}\left(\sum_{r=1}^{2 n} x_{r} e_{i_{r}}^{*} \otimes e_{j_{r}}\right), \quad \text { by Lemma } 2.1 \\
& =\sum_{\sigma, \delta} \varepsilon_{\sigma} \varepsilon_{\delta} a_{\sigma(1) \delta(1) \sigma(2) \delta(2)} \cdots a_{\sigma(2 n-1) \delta(2 n-1) \sigma(2 n) \delta(2 n)},
\end{aligned}
$$

which proves Lemma 2.3.

Now suppose that we are given a nondegenerate bilinear form $B$ in $V$. Then $B$ induces a linear map $\varphi$ from $V \otimes V$ into the field $R$ such that

$$
B(v, w)=\varphi(v \otimes w), \quad v, w \in V .
$$

Also the bilinear from $B$ induces an isomorphism of $V$ onto its dual $V^{*}$. Thus we have $V \otimes V \approx V^{*} \otimes V \approx \operatorname{Hom}(V, V)$. Suppose that under this isomorphism the element $x$ of $V \otimes V$ corresponds to the element $\tilde{\varphi}(x)$ of $\operatorname{Hom}(V, V)$. Then it is a trivial fact that

$$
\varphi(x)=\operatorname{Tr} \tilde{\varphi}(x) \quad \text { for all } x \in V \otimes V
$$

\section{Commutation formulas for covariant differentiation}

Our next considerations are with respect to the manifold $X$. Let $U$ be an open subset of $X$ such that $U$ is diffeomorphic to an open subset of $\boldsymbol{R}^{d}$. We shall denote the coordinate functions by $y_{1}, \cdots, y_{d}$, and the tangent space and the cotangent space at a point $y$ of $U$ by $T_{y}(X)$ and $T_{y}^{*}(X)$ respectively.

Let $A \in C^{\infty}\left(U, T(X) \otimes T^{*}(X)\right)$. Then for each point $y$ of $U, A(y)$ is an endomorphism of $T_{y}^{*}(X)$ and hence defines an endomorphism $D^{p} A(y)$ of $\wedge^{p} T_{y}^{*}(X)$. Thus we get a linear operator $D^{p} A$ from the space of exterior $p$-forms defined on $U$ into itself:

$$
\left(D^{p} A(\alpha)\right)(y)=\left(D^{p} A(y)\right)(\alpha(y)),
$$


where $\alpha$ is an exterior $p$-form defined on $U$, and $y \in U$.

If $\alpha$ is a $C^{\infty}$-form on $U$, then so is $D^{p} A(\alpha)$. Similarly, if $A \in C^{\infty}(U, T(X) \otimes$ $\left.T^{*}(X) \otimes T(X) \otimes T^{*}(X)\right)$, we have a linear operator $D^{p} A$ from $C^{\infty}\left(U, \wedge^{p} T^{*}(X)\right)$ into itself.

Curvature tensor field $R$ is a tensor field of type (1.3). But for all $y \in X$, the Riemannian metric induces a natural isomorphism of $T_{y}^{*}(X)$ onto $T_{y}(X)$ and hence an isomorphism of $T_{y}(X) \otimes T_{y}^{*}(X) \otimes T_{y}^{*}(X) \otimes T_{y}^{*}(X)$ onto $T_{y}(X)$ $\otimes T_{y}^{*}(X) \otimes T_{y}(X) \otimes T_{y}^{*}(X)$. Under this isomophism, $R$ can be considered as a tensor field of type $(2,2)$ and thus defines a linear operator $D^{p} R$ from $C^{\infty}\left(U, \wedge^{p} T^{*}(X)\right)$ into itself.

Lemma 3.1. The Laplace operator $\Delta$ acting on p-forms is given by

$$
\Delta=\sum_{i, j} g^{i j} \nabla_{j} \circ \nabla_{i}-\sum_{i, j, a} g^{i j} \Gamma_{i j}^{a} \nabla_{a}+D^{p} R,
$$

where the matrix $\left(g^{i j}\right)$ is the inverse of the matrix $\left(g_{i j}\right), g_{i j}$ being the components of the Riemannian metric, and $\Gamma_{i j}^{k}$ are the Christoffel symbols defined by

$$
\Gamma_{i j}^{k}=\frac{1}{2} \sum_{a} g^{k a}\left(\frac{\partial g_{a i}}{\partial y_{i}}+\frac{\partial g_{a j}}{\partial y_{i}}-\frac{\partial g_{i j}}{\partial y_{a}}\right)
$$

Proof. This lemma is well known; see [4], [5].

Lemma 3.2. Let $X_{1}, \cdots, X_{m}$ be $C^{\infty}$-vector fields on the open set $U$, $A \in C^{\infty}\left(U, T(X) \otimes T^{*}(X)\right)$, and the operators $\nabla_{X_{1}}, \cdots, \nabla_{X_{m}}$ of covariant derivation (with respect to Levi-Civita connection) be denoted respectively by $\nabla_{1}, \cdots, \nabla_{m}$. Then we have the following commutation relation:

$$
\begin{aligned}
& \nabla_{1} \circ \cdots \circ \nabla_{m} \circ D^{p}(A)=D^{p} A \circ \nabla_{1} \circ \cdots \circ \nabla_{m} \\
& \quad+\sum_{k=1}^{m} \sum_{\substack{\sigma(1)<\cdots<<(k) \\
\sigma(k+1)<\cdots<\sigma(m)}} D^{p}\left(\nabla_{\sigma(1)} \circ \cdots \circ \nabla_{\sigma(k)}(A)\right) \circ \nabla_{\sigma(k+1)} \circ \cdots \circ \nabla_{\sigma(m)},
\end{aligned}
$$

where the second sum on the right hand side runs over all permutations $\sigma$ of $\{1, \cdots, m\}$ such that $\sigma(1)<\cdots<\sigma(k)$ and $\sigma(k+1)<\cdots<\sigma(m)$.

Proof. We shall prove the lemma by induction on $m$. First we prove the lemma for $m=1$, that is,

$$
\nabla_{1} \circ D^{p} A(\alpha)=D^{p} A \circ \nabla_{1}(\alpha)+D^{p}\left(\nabla_{1}(A)\right)(\alpha), \quad \alpha \in C^{\infty}\left(U, \wedge^{p} T^{*}(X)\right) .
$$

For $p=1$, the above formula can be verified by direct calculations. But noting that $\nabla_{1}$ and $D^{p}(A)$ are derivations, one easily observes that if the above formula is true for $\alpha^{p}, \alpha^{q}, \alpha^{p} \in C^{\infty}\left(U, \wedge^{p} T^{*}(X)\right)$ and $\alpha^{q} \in C^{\infty}\left(U, \wedge^{q} T^{*}(X)\right)$, then it also holds for $\alpha=\alpha^{p} \wedge \alpha^{q}$. Hence by induction on $p$, it follows that (3.2) holds for $m=1$.

Let $r$ be a positive integer, and suppose that the lemma has been proved for 
$m \leq r$. Then we complete the proof of the lemma by the fact that (3.2) holds for $m=r+1$ since

$$
\begin{aligned}
& \nabla_{1} \circ \cdots \circ \nabla_{r+1} \circ D^{p} A \\
& =\nabla_{1} \circ \cdots \circ \nabla_{r} \circ D^{p} A \circ \nabla_{r+1}+\nabla_{1} \circ \ldots \circ \nabla_{r} \circ D^{p}\left(\nabla_{r+1}(A)\right) \\
& =D^{p} A \circ \nabla_{1} \circ \cdots \circ \nabla_{r} \circ \nabla_{r+1} \\
& +\left(\sum_{k=1}^{r} \sum_{\substack{\delta(1)<\cdots<\delta(k) \\
\delta(k+1)<\cdots<\delta(r)}} D^{p}\left(\nabla_{\delta(1)} \circ \cdots \circ \nabla_{\delta(k)}(A)\right) \circ \nabla_{\delta(k+1)} \circ \cdots \circ \nabla_{\delta(r)}\right) \circ \nabla_{r+1} \\
& +\sum_{k=0}^{r} \sum_{\substack{\rho(1)<\cdots<\rho(k) \\
\rho(k+1)<\cdots<\rho(r)}} D^{p}\left(\nabla_{\rho(1)} \circ \cdots \circ \nabla_{\rho(k)} \circ \nabla_{r+1}(A)\right) \circ \nabla_{\rho(k+1)} \circ \cdots \circ \nabla_{\rho(r)} \\
& \text { by the induction hypothesis } \\
& =D^{p} A \circ \nabla_{1} \circ \cdots \circ \nabla_{r} \circ \nabla_{r+1}+\sum_{k=1}^{r+1} \sum_{\substack{\sigma(1)<\cdots<\sigma(k) \\
\sigma(k+1)<\cdots<\sigma(r+1)}} D^{p}\left(\nabla_{\sigma(1)} \circ \cdots \circ \nabla_{\sigma(k)}(A)\right) \\
& \circ \nabla_{\sigma(k+1)} \circ \cdots \circ \nabla_{\sigma(r+1)} .
\end{aligned}
$$

Lemma 3.3. Let $X_{1}, \cdots, X_{m}$ be $C^{\infty}$-vector fields on the open set $U$, $A \in C^{\infty}\left(U, T(X) \otimes T^{*}(X) \otimes T(X) \otimes T^{*}(X)\right)$, and the operators $\nabla_{X_{1}}, \cdots, \nabla_{X_{m}}$ of covariant derivation be denoted respectively by $\nabla_{1}, \cdots, \nabla_{m}$. Then the formula (2) holds.

Proof. It is enough to prove the lemma when $A=B \otimes C, B \in C^{\infty}(U$, $\left.T(X) \otimes T^{*}(X)\right), C \in C^{\infty}\left(U, T(X) \otimes T^{*}(X)\right)$. Then we have

$$
\begin{aligned}
\nabla_{1} \circ & D^{p} A=\nabla_{1} \circ D^{p} B \circ D^{p} C \\
& =D^{p} B \circ \nabla_{1} \circ D^{p} C+D^{p}\left(\nabla_{1}(B)\right) \circ D^{p} C, \quad \text { by Lemma } 3.2 \\
& =D^{p} B \circ D^{p} C \circ \nabla_{1}+D^{p} B \circ D^{p}\left(\nabla_{1}(C)\right)+D^{p}\left(\nabla_{1}(B) \otimes C\right), \text { by Lemma } 3.2 \\
& =D^{p}(B \otimes C) \circ \nabla_{1}+D^{p}\left(B \otimes \nabla_{1}(C)\right)+D^{p}\left(\nabla_{1}(B) \otimes C\right) \\
& =D^{p}(A) \circ \nabla_{1}+D^{p}\left(\nabla_{1}(B \otimes C)\right) \\
& =D^{p} A \circ \nabla_{1}+D^{p}\left(\nabla_{1}(A)\right) .
\end{aligned}
$$

Thus we have proved the lemma for $m=1$, and the lemma follows for all $m$ by arguing inductively on $m$ as in the proof of Lemma 3.2.

Let $R=\sum R_{j k l}^{i}\left(\partial / \partial y_{i}\right) \otimes d y_{j} \otimes d y_{k} \otimes d y_{l}$ be the curvature tensor field, and for any two vector fields $X, Y$ on $U$ let $R(X, Y)$ be the tensor field of type $(1,1)$ defined by

$$
R(X, Y)=\sum R_{j k l}^{i} X\left(d y_{k}\right) Y\left(d y_{l}\right) \frac{\partial}{\partial y_{i}} \otimes d y_{j}
$$

Lemma 3.4. Let $X, X_{1}, \cdots, X_{m}$ be $C^{\infty}$-vector fields defined on $U$, and the 
operators $\nabla_{X_{1}}, \cdots, \nabla_{X_{m}}$ of covariant derivations be denoted respectively by $\nabla_{1}, \cdots, \nabla_{m}$. Then

$$
\begin{aligned}
& \nabla_{1} \circ \cdots \circ \nabla_{m} \circ \nabla_{X}=\nabla_{X} \circ \nabla_{1} \circ \cdots \circ \nabla_{m} \\
& +\sum_{j=0}^{m-1} \sum_{\substack{\sigma(1)<\cdots<j(j+2)<\cdots<\sigma(m) \\
\sigma(j+1)}} D^{p}\left(\nabla_{\sigma(1)} \circ \cdots \circ \nabla_{\sigma(j)} R\left(X, X_{\sigma(j+1)}\right)\right) \\
& \circ \nabla_{\sigma(j+2)} \circ \cdots \circ \nabla_{\sigma(m)} \\
& +\sum_{i=1}^{m} \nabla_{1} \circ \cdots \circ \nabla_{i-1} \circ \nabla_{\left[X_{i}, X\right]} \circ \nabla_{i+1} \circ \cdots \circ \nabla_{m} \text {. }
\end{aligned}
$$

Proof. For $m=1$, formula (3.3) for forms of degree one is just the definition of curvature tensor, and for forms of degree $p \geq 1$ it follows easily by arguing inductively on $p$ and using the derivation properties of the operators. Then proof of Lemma 3.4 can be completed by induction on $m$ as in the proof of Lemma 3.2.

\section{Construction of a parametrix and the fundamental solution}

We shall first introduce some notation. Let $U$ be an open subset of $X$, and $\alpha(x, y)$ a $C^{\infty}(p, p)$ form on $U \times U$. Then $\alpha \in C^{\infty}\left(U \times U, \wedge^{p} T^{*}(X) \otimes \wedge^{p} T^{*}(X)\right)$. Since for all $x \in X$ the Riemannian metric induces a natural isomorphism of $\wedge^{p} T_{x}^{*}(X)$ onto the dual of $\wedge^{p} T_{x}^{*}(X)$, there is a natural identification of $\wedge^{p} T^{*}(X) \otimes \wedge^{p} T^{*}(X)$ with Hom $\left(\wedge^{p} T^{*}(X), \wedge^{p} T^{*}(X)\right)$, and therefore we can regard $\alpha$ as an element of $C^{\infty}\left(U \times U\right.$, Hom $\left.\left(\wedge^{p} T^{*}(X), \wedge^{p} T^{*}(X)\right)\right)$. Let $x \in U$ and $v \in \wedge^{p} T_{x}^{*}(X)$. Then $\alpha(x, y)(v)$ is a smooth $p$-form defined on $U$, and will be denoted by $\alpha(v, y)$.

For $p$-forms we construct a parametrix $H_{N}^{p}(t, x, y)$ in a sufficiently small neighbourhood of the diagonal in $X \times X, t>0$, as follows:

$$
H_{N}^{p}(t, x, y)=\frac{\exp \left(-r^{2} /(4 t)\right)}{(4 \pi t)^{d / 2}}\left(\sum_{i=0}^{N} t^{i} U^{i, p}(x, y)\right)
$$

where $r$ is the geodesic distance between $x, y$, and the smooth $(p, p)$ forms $U^{i, p}(x, y)$ are to be determined such that $U^{0, p}(x, x)$ is the identity endomorphism of $\wedge^{p} T_{x}^{*}(X)$ and

$$
\left(\frac{\partial}{\partial t}-\Delta_{y}\right) H_{N}^{p}(t, x, y)=-\frac{\exp \left(-r^{2} /(4 t)\right)}{(4 \pi t)^{d / 2}} t^{N} \Delta_{y} U^{N, p}(x, y) .
$$

The integer $N$ is to be chosen larger than $d / 2$. These conditions determine the double $(p, p)$ forms $U^{i, p}(x, y)$ uniquely in a sufficiently small neighbourhood of the diagonal as we shall see now.

Fix an arbitrary point $x$ of $X$ and introduce normal coordinates in an open 
neighbourhood $U$ of $x$ such that $g_{i j}(x)=\delta_{i j}$ and $x$ has the coordinates $(0, \cdots, 0)$. Let $F(r(x, y))$ be a function of $y$ depending only on the geodesic distance of $y$ from the point $x$, and $\alpha$ be a $C^{\infty} p$-form defined on $U$. It is well known that (see for instance [3])

$\Delta_{y}(F(r) \alpha)$

$=\left(\frac{d^{2} F}{d r^{2}}(r)+\frac{d-1}{r} \frac{d F}{d r}(r)+\frac{1}{2 g} \frac{d g}{d r} \frac{d F}{d r}(r)\right) \alpha+\frac{2}{r} \frac{d F}{d r}(r) \nabla_{r \frac{d}{d r}}(\alpha)+F(r) \Delta \alpha$,

where $d / d r$ denotes the differentiation along the geodesic, and $g(y)=\operatorname{det}\left(g_{i j}(y)\right)$. Thus

$$
\begin{aligned}
& \Delta_{y}\left(\left(\exp \left(-r^{2} / 4 t\right)\right) \alpha\right) \\
& \quad=\exp \left(-\frac{r^{2}}{4 t}\right)\left\{\left(\frac{r^{2}}{4 t^{2}}-\frac{1}{2 t}-\frac{d-1}{2 t}-\frac{r}{4 g t} \frac{d g}{d r}\right) \alpha-\frac{1}{t} \nabla_{r \frac{d}{d r}} \alpha+\Delta \alpha\right\},
\end{aligned}
$$

and therefore

$$
\begin{aligned}
\left(\frac{\partial}{\partial t}-\Delta_{y}\right) H_{N}^{p}(t, x, y) & \\
= & \frac{\exp \left(-r^{2} /(4 t)\right)}{(4 \pi t)^{d / 2}} \sum_{i=0}^{N}\left\{\left(\frac{r^{2}}{4 t^{2}}+\frac{i-d / 2}{t}-\frac{r^{2}}{4 t^{2}}+\frac{1}{2 t}+\frac{d-1}{2 t}\right.\right. \\
& \left.\left.\quad+\frac{r}{4 g t} \frac{d g}{d r}\right) t^{i} U^{i, p}(x, y)+t^{i-1} \nabla_{r \frac{d}{d r}} U^{i, p}(x, y)-t^{i} \Delta_{y} U^{i, p}(x, y)\right\} .
\end{aligned}
$$

Equating the coefficient of $\frac{\exp \left(-r^{2} /(4 t)\right)}{(4 \pi t)^{d / 2}} t^{i-1}$ in $\left(\frac{\partial}{\partial t}-\Delta_{y}\right) H_{N}^{p}(t, x, y)$ to zero, we get

$$
\left(i+\frac{r}{4 g} \frac{d g}{d r}\right) U^{i, p}(x, y)+\nabla_{r \frac{d}{d r}} U^{i, p}(x, y)-\Delta_{y} U^{i-1, p}(x, y)=0,
$$

that is,

$$
\nabla_{r \frac{d}{d r}} U^{i, p}(x, y)+\left(i+\frac{r}{4 g} \frac{d g}{d r}\right) U^{i, p}=\Delta_{y} U^{i-1, p}(x, y)
$$

Fix an arbitrary vector $v \in \wedge^{p} T_{x}^{*}(X)$. Then we shall show that in the open set $U$ the equations

$$
\nabla_{r \frac{d}{d r}} U^{i, p}(v, y)+\left(i+\frac{r}{4 g} \frac{d g}{d r}\right) U^{i, p}(v, y)=\Delta_{y} U^{i-1}(v, y), 0 \leq i \leq N
$$


have unique solutions with the condition $U^{0, p}(v, x)=v$; of course $U^{-1, p}(x, y) \equiv 0$.

We rewrite equation (4.4) in the form

$$
\nabla_{r \frac{d}{d r}}\left(r^{i} g^{1 / 4} U^{i, p}(v, y)\right)=r^{i} g^{1 / 4} \Delta_{y} U^{i-1, p}(v, y) .
$$

Let $y$ be an arbitrary point of $U$, and $u_{y}(t), 0 \leq t \leq r(x, y)$, be the geodesic curve joining the points $x$ and $y$. The curve $u_{y}(t)$ defines, with respect to the Riemannian connection, an isomorphism $T_{y, t_{0}}$ of $\wedge^{p} T_{u_{y}\left(t_{0}\right)}^{*}(X)$ onto $\wedge^{p} T_{y}^{*}(X)$, $0 \leq t_{0} \leq r(x, y)$. Let $U^{0, p}(v, y)=g^{-1 / 4}(y) T_{y, 0}(v)$. Then $U^{0, p}(v, x)=v$, and equation (4.5) is satisfied for $i=0$. Let $m$ be a positive integer, and suppose that for $i<m$ we have determined the forms $U^{i, p}(v, y)$ satisfying equation (4.5). Then we define $U^{m, p}(v, y)$ as

$$
\begin{aligned}
& U^{m, p}(v, y) \\
& =\frac{1}{(r(x, y))^{m} g^{1 / 4}(y)} \int_{0}^{r(x, y)}\left(r\left(x, u_{y}(t)\right)\right)^{m-1} g^{1 / 4}\left(u_{y}(t)\right) T_{y, t}\left(\Delta_{y} U^{m-1}\left(v, u_{y}(t)\right)\right) d t .
\end{aligned}
$$

It can easily be seen that $U^{m, p}(v, y)$ is a $C^{\infty}$-form and satisfies equation (4.5) for $i=m$.

To prove the uniqueness, we first observe that equation (4.2) implies $i U^{i, p}(v, x)=\left(\Delta_{y} U^{i-1, p}(v, y)\right)(v, x)$. Therefore it sufficies to prove that any $C^{\infty}$ solution $\alpha$ of $\nabla_{r \frac{d}{d r}}(\alpha)=0$ satisfying the initial condition $\alpha(x)=0$ vanishes identically. But this is obvious because $\nabla_{r \frac{d}{d r}}(\alpha)=0$ implies that for all $y \in U, \alpha$ is invariant under the parallel displacements along the geodesic joining the points $x$ and $y$. Thus we can construct the parametrix $H_{N}^{p}(t, x, y)$ in a sufficiently small neighbourhood $W$ of the diagonal in $X \times X$. Let $W^{\prime}$ be an open neighbourhood of the diagonal such that the closure of $W^{\prime}$ is contained in the interior of $W$, and $\phi(x, y)$ be a $C^{\infty}$-function on $X \times X$ such that $\phi(x, y)$ is zero-outside $W^{\prime}$ and is one in a neighbourhood of the diagonal. Then we define

$$
\begin{aligned}
& G_{N}^{p}(t, x, y)=\psi(x, y) H_{N}^{p}(t, x, y) \\
& K_{N}^{p}(t, x, y)=\left(\frac{\partial}{\partial t}-\Delta_{y}\right) G_{N}^{p}(t, x, y) .
\end{aligned}
$$

The double form $G_{N}^{p}(t, x, y)$ is a parametrix in the large, and is a $C^{\infty}$-double form. It can be verified that for any smooth $p$-form $\varphi(t, x)$ one has

$$
\underset{t \rightarrow 0}{L t} \int_{X} G_{N}^{p}(t, x, y) \wedge * \varphi(t, x)=\varphi(0, y),
$$

where in the limit $t$ tends to zero only through positive values. For the sake of 
simplicity of notation we shall fix $p, N$ and denote $K_{N}^{p}(t, x, y)$ by $K(t, x, y)$.

We now complete the construction of the fundamental solution of the operator $\frac{\partial}{\partial t}-\Delta$. We first observe that if $M, N_{1}, N_{2}$ are vector spaces and there is given an inner product in $M$, then there is a natural map $\tau:\left(M \otimes N_{1}\right) \times\left(M \otimes N_{2}\right)$ $\rightarrow N_{1} \otimes N_{2}$ such that

$\tau\left(\left(m \otimes n_{1}\right),\left(m^{\prime} \otimes n_{2}\right)\right)=\left\langle m, m^{\prime}\right\rangle n_{1} \otimes n_{2}, \quad$ for $m, m^{\prime} \in M, n_{1} \in N_{1}, n_{2} \in N_{2}$,

$\langle$,$\rangle being the inner product in M$. We shall denote the element $\tau(x, y)$ for $x \in M \otimes N_{1}$ and $y \in M \otimes N_{2}$ by the symbol $(x, y)$ itself.

Inductively we now define

$$
\begin{aligned}
K^{0}(t, x, y) & =K(t, x, y), \\
K^{m}(t, x, y) & =\int_{0}^{t} d s \int_{X}\left(K^{m-1}(s, x, z), K(t-s, z, y)\right) *_{z} 1, \quad m \geq 1,
\end{aligned}
$$

$*_{z} 1$ being the volume element. Since $X$ is compact, there exist finitely many open sets $V_{1}, \cdots, V_{q}$ and $U_{1}, \cdots, U_{q}$ such that $\bar{V}_{r} \subset U_{r}, U_{r}$ is diffeomorphic to $\boldsymbol{R}^{d}, 1 \leq r \leq q$, and $X=\bigcup_{r=1}^{q} V_{r}$. Choose a partition of unity $\left\{\varphi_{r}\right\}_{1 \leq r \leq q}$ relative to the open covering $\left\{V_{r}\right\}_{1 \leq r \leq q}$, and let $\phi_{r}$ be $C^{\infty}$-functions which are 1 on $V_{r}$ and have supports contained in $U_{r}$. For any double form $L(x, y)$ define

$$
L_{i, j}(x, y)=\phi_{i}(x) \phi_{j}(y) L(x, y)
$$

and for a form

$$
L(x, y)=\sum_{\substack{i_{1}<i<<i p \\ j_{1}<\cdots<j p}} L_{i_{1} \cdots i_{p}, j_{1} \cdots j_{p}} d x_{i_{1}} \wedge \cdots \wedge d x_{i_{p}} \otimes d y_{j_{1}} \wedge \cdots \wedge d y_{j_{p}}
$$

with support contained in $U_{i} \times U_{j}$ define

$$
\|L\|_{i, j}=\sum_{\substack{i_{1}<\cdots<i_{p} \\ j_{1}<\cdots<j_{p}}} \operatorname{Sup}_{x \in U_{i}, y \in U j}\left|L_{i_{1} \cdots i_{p}, j_{1} \cdots j_{p}}\right| .
$$

Then by (4.2), $\left\|K_{i, j}^{0}\right\|_{i, j} \leq M t^{N-d / 2}$ for some constant $M$. Suppose we have proved that

$$
\left\|K_{i, j}^{m-1}\right\|_{i, j} \leq(C M)^{m} t^{m(N-d / 2)+m-1} \frac{(\Gamma(N-d / 2+1))^{m}}{\Gamma(m(N-d / 2)+m)},
$$

with a constant $C$. Then 


$$
\begin{aligned}
K_{i, j}^{m}(t, x, y) & =\int_{0}^{t} d s \int_{X}\left(\phi_{i}(x) \sum_{r=1}^{q} \varphi_{r}(z) K^{m-1}(s, x, z), \phi_{j}(y) K(t-s, z, y)\right) *_{z} 1 \\
& =\sum_{r=1}^{q} \int_{0}^{t} d s \int_{X}\left(\phi_{i}(x) \varphi_{r}(z) K^{m-1}(s, x, z), K_{r, j}(t-s, z, y)\right) *_{z} 1
\end{aligned}
$$

Therefore

$$
\begin{aligned}
\left\|K_{i, j}^{m}(t, x, y)\right\| \leq & C_{1} \cdot(C M)^{m} \frac{(\Gamma(N-d / 2+1))^{m}}{\Gamma(m(N-d / 2)+m)} M \\
& \cdot \int_{0}^{t} s^{m(N-d / 2)+m-1}(t-s)^{N-d / 2} d s,
\end{aligned}
$$

where $C_{1}$ is a constant independent of $m$, so that, by choosing $C>C_{1}$,

$$
\begin{aligned}
& \left\|K_{i, j}^{m}(t, x, y)\right\| \\
& \quad \leq(C M)^{m+1} t^{(m+1)(N-d / 2)+m} \frac{\Gamma(N-d / 2+1)^{m+1}}{\Gamma((m+1)(N-d / 2)+m+1)}
\end{aligned}
$$

We set

$$
\begin{aligned}
& e^{p}(t, x, y) \\
& =G_{N}^{p}(t, x, y)+\sum_{m \geq 0}(-1)^{m+1} \int_{0}^{t} d s \int_{X}\left(K^{m}(s, x, z), G_{N}^{p}(t-s, z, y)\right) *_{z} 1 .
\end{aligned}
$$

On account of the estimate (4.6) and the similar ones which we can obtain by introducing partial derivatives also in above norms, the series on the right hand side of (4.7) converges nicely to a double form. Hence $e^{p}(t, x, y)$ is a $C^{\infty}-(p, p)$ form, and we have

$$
\begin{aligned}
\left(\frac{\partial}{\partial t}-\Delta_{y}\right)\left(e^{p}(t, x, y)\right) & =K(t, x, y)+\sum_{m \geq 0}(-1)^{m+1}\left(K^{m}(t, x, y)+K^{m+1}(t, x, y)\right) \\
& =K(t, x, y)-K(t, x, y)=0 .
\end{aligned}
$$

The double form $e^{p}(t, x, y)$ is the fundamental solution for the heat operator acting on $p$-forms.

From now on we shall fix a point $x$ of the manifold $X$. We introduce normal coordinates in an open neighbourhood $U$ of $x$ such that $g_{i j}(x)=\delta_{i j}$. By the estimate (4.6) we have

$$
\sum_{m=0}^{\infty}(-1)^{m+1} \int_{0}^{t} d s \int_{X}\left(K^{m}(s, x, z), G_{N}^{p}(t-s, z, x)\right) *_{z} 1
$$




$$
\begin{aligned}
& =0 \int_{0}^{t} d s \int_{U} \exp \left(-\frac{(r(z, x))^{2}}{4(t-s)}\right)(t-s)^{-d / 2} s^{N-d / 2} *_{z} 1, \quad t \downarrow 0 \\
& =0 \int_{0}^{t} d s \int_{0}^{\infty} \exp \left(-\frac{r^{2}}{4(t-s)}\right)(t-s)^{-d / 2} s^{N-d / 2} r^{d-1} d r \\
& =0 \int_{0}^{t} d s \int_{0}^{\infty} e^{-u 2}(t-s)^{-d / 2} s^{N-d / 2} u^{d-1}(t-s)^{d / 2} d u, \\
& \text { by making the substitution } r \mapsto 2 u(t-s)^{1 / 2} \\
& =0 \int_{0}^{t} s^{N-d / 2} d s=0\left(t^{N-d / 2+1}\right), \quad t \downarrow 0 .
\end{aligned}
$$

Therefore by (4.7) we get

$$
\begin{aligned}
\left(\operatorname{Tr} e^{p}\right)(t, x, x) & =\left(\operatorname{Tr} G_{N}^{p}\right)(t, x, x)+0\left(t^{N-d / 2+1}\right) \\
& =\left(\operatorname{Tr} H_{N}^{p}\right)(t, x, x)+0\left(t^{N-d / 2+1}\right) \\
& =(4 \pi)^{-d / 2} \sum_{i=0}^{N} t^{i} \operatorname{Tr} U^{i, p}(x, x)+0\left(t^{N-d / 2+1}\right), \quad \text { by (4.1). }
\end{aligned}
$$

The double forms $U^{i, p}(x, y)$ satisfy equation (4.1). Since $U^{0, p}(x, x)$ is the identity endomorphism of $\wedge^{p} T_{x}^{*}$, we have

$$
\begin{aligned}
& \left(\operatorname{Tr} e^{p}\right)(t, x, x) \\
& \quad=(4 \pi t)^{-d / 2}\left\{\left(\begin{array}{l}
d \\
p
\end{array}\right)+\sum_{i=1}^{N} t^{i} \operatorname{Tr} U^{i, p}(x, x)\right\}+0\left(t^{N-d / 2+1}\right), \quad t \downarrow 0 .
\end{aligned}
$$

\section{Proof of the theorem}

Suppose that the dimension $d$ of the manifold is an even integer $2 n$. In view of (4.8), the theorem is an immediate consequence of

Lemma 5.1. $(4 \pi)^{-n} \sum_{p=0}^{d}(-1)^{p} \operatorname{Tr} U^{i, p}(x, x)= \begin{cases}0 & \text { for } i<n, \\ C & \text { for } i=n,\end{cases}$ $C$ being Chern's polynomial at the point $x$ :

$$
C=\frac{1}{2^{3 n} \pi^{n} n !} \sum \varepsilon_{\sigma} \varepsilon_{\delta} R_{\sigma(1) \sigma(2) \delta(1) \delta(2)} \cdots R_{\sigma(2 n-1) \sigma(2 n) \delta(2 n-1) \delta(2 n)},
$$

where the sum runs over all the permutations $\sigma, \delta$ of $1, \cdots, 2 n, \varepsilon_{\sigma}$ and $\varepsilon_{\delta}$ are the signatures of the permutations $\sigma$ and $\delta$ respectively, and $R_{i j k l}$ are the components of the curvature tensor (see [1]):

$$
R=\sum R_{j i k l} \frac{\partial}{\partial y_{i}} \otimes d y_{j} \otimes d y_{k} \otimes d y_{l}
$$


For the proof of Lemma 5.1, we will need the following

Main lemma 5.2. Let $l_{1}, l_{2}, l_{3}, i$ be nonnegative integers such that one of the following conditions is satisfied;

( $\left.C_{1}\right) \quad l_{3}>0$ and $l_{1}+2 l_{2}+l_{3}+2 i \leq d$,

$\left(C_{2}\right) \quad l_{1}+2 l_{2}+l_{3}+2 i<d$.

Let $\sigma$ be a permutation of $\left\{1, \cdots, l_{1}+l_{2}\right\}$, and $B_{\sigma(1)}, \cdots, B_{\sigma\left(l_{1}\right)}$ and $B_{\sigma\left(l_{1}+1\right)}$, $\cdots, B_{\sigma\left(l_{1}+l_{2}\right)}$ be arbitrary $C^{\infty}$-sections of $T(X) \otimes T^{*}(X)$ and $T(X) \otimes T^{*}(X)$ $\otimes T(X) \otimes T^{*}(X)$ defined over $U$ respectively. Let $\left\{j_{1}, \cdots, j_{l_{3}}\right\}$ be a sequence such that each $j_{r}$ is a positive integer between 1 and $d$, and the operators $\nabla_{\frac{\partial}{\partial y_{j_{1}}}}, \nabla_{\frac{\partial}{\partial y_{j_{2}}}}, \cdots$ be denoted respectively by $\nabla_{j_{1}}, \nabla_{j_{2}}, \ldots$ Then

$$
\sum_{p=0}^{d}(-1)^{p} \operatorname{Tr}\left[D^{p} B_{1} \circ \cdots \circ D^{p} B_{l_{1}+l_{2}} \circ \nabla_{j_{1}} \circ \cdots \circ \nabla_{j_{3}}\left(U^{i, p}(x, y)\right)\right](x, x)=0,
$$

where all the operators act with respect to the variable $y$.

Proof of Lemma 5.2. We shall prove the lemma by induction on $i$ and $l_{3}$. Let $s$ be a nonnegative integer, and suppose that the lemma has been proved whenever $i<s$ and the integers $l_{1}, l_{2}, l_{3}, i$ satisfy at least one of the conditions $\left(C_{1}\right)$ and $\left(C_{2}\right)$. We shall prove the lemma for $i=s$.

Let $B^{p}$ be the operator defined by

$$
B^{p}=D^{p} B_{1} \circ \cdots \circ D^{p} B_{l_{1}+l_{2}} \circ \nabla_{j_{1}} \circ \cdots \circ \nabla_{j_{l_{3}}} .
$$

$U^{s, p}(x, y)$ satisfies the differential equation

$$
\begin{aligned}
& \nabla_{r \frac{d}{d r}} U^{s, p}(x, y)+\left(s+\frac{r}{4 g} \frac{d g}{d r}\right) U^{s, p}(x, y)=\Delta_{y} U^{s-1, p}(x, y) \\
& =\left(\sum_{k, j} g^{k j} \nabla_{j} \circ \nabla_{k}-\sum_{k, j, a} g^{k j} \Gamma_{k j}^{a} \nabla_{a}+D^{p} R\right) U^{s-1, p}(x, y), \quad \text { by (1.2). }
\end{aligned}
$$

First suppose that $l_{3}=0$. If $s=0$, then the result follows from Lemma 2.2. Therefore we can assume that $s>0$. Since the double form $\nabla_{r_{\frac{d}{d r}}} U^{s, p}$ is zero at $(x, x)$, we have

$$
\sum_{p=0}^{d}(-1)^{p} \operatorname{Tr}\left[B^{p}\left(\nabla_{r \frac{d}{d r}} U^{s, p}\right)\right](x, x)=0
$$

By first applying the operator $B^{p}$ to both sides of equation (5.1) and then taking trace we thus obtain

$$
s \sum_{p=0}^{d}(-1)^{p} \operatorname{Tr}\left[B^{p}\left(U^{s, p}(x, y)\right](x, x)\right.
$$




$$
\begin{aligned}
= & \sum_{k, j} g^{k j} \sum_{p=0}^{d}(-1)^{p} \operatorname{Tr}\left[B^{p} \circ \nabla_{j} \circ \nabla_{k}\left(U^{s-1, p}(x, y)\right)\right](x, x) \\
& -\sum_{k, j, a} g^{k j} \Gamma_{k j}^{a} \sum_{p=0}^{d}(-1)^{p} \operatorname{Tr}\left[B^{p} \circ \nabla_{a}\left(U^{s-1, p}(x, y)\right)\right](x, x) \\
& +\sum_{q=0}^{d}(-1)^{p} \operatorname{Tr}\left[B^{p} \circ D^{p} R\left(U^{s-1, p}(x, y)\right)\right](x, x) .
\end{aligned}
$$

Since each term on the right hand side of (5.2) is zero by the induction hypothesis, we have

$$
\sum_{p=0}^{d}(-1)^{p} \operatorname{Tr}\left[B^{p}\left(U^{s, p}(x, y)\right)\right](x, x)=0
$$

Now suppose that $l_{3}>0$ and the result has been proved for smaller values of $l_{3}$. For first applying the operator $B^{p}$ to both sides of equation (5.1) and then taking the trace, we first note that $\left[\frac{\partial}{\partial y_{i}}, r \frac{d}{d r}\right]=\frac{\partial}{\partial y_{i}}$. In fact, $r \frac{d}{d r}=$ $\sum\left(y_{j}-x_{j}\right) \frac{\partial}{\partial y_{j}}$ so that

$$
\frac{\partial}{\partial y_{i}}\left(r \frac{d}{d r}\right)=\sum\left(y_{j}-x_{j}\right) \frac{\partial}{\partial y_{i}} \frac{\partial}{\partial y_{j}}+\frac{\partial}{\partial y_{i}}=r \frac{d}{d r} \frac{\partial}{\partial y_{i}}+\frac{\partial}{\partial y_{i}} .
$$

Therefore by Lemma 3.4, $B^{p} \circ \nabla_{r \frac{d}{d r}}$ equals $D^{p} B_{1} \circ \ldots \circ D^{p} B_{l_{1}+l_{2}} \circ \nabla_{r \frac{d}{d r}} \circ \nabla_{j_{1}} \circ$ $\ldots \circ \nabla_{j_{l_{3}}}+l_{3} B^{p}$ plus a sum of operators for each of which the induction hypothesis for $l_{3}$ is satisfied (with $i=s$ ). Also the form $\nabla_{r_{\frac{d}{d r}}} \circ \nabla_{j_{1}} \circ \cdots \circ \nabla_{j_{l_{3}}}\left(U^{s, p}(x, y)\right.$ ) is zero at $(x, x)$. Hence we have

$$
\sum_{p=0}^{d}(-1)^{p} \operatorname{Tr}\left[B^{p} \circ \nabla r \frac{d}{d r}\left(U^{s, p}\right)\right](x, x)=l_{3} \sum_{p=0}^{d}(-1)^{p} \operatorname{Tr}\left[B^{p}\left(U^{s, p}\right)\right](x, x),
$$

and also

$$
\begin{aligned}
& B^{p}\left(\left(s+\frac{r}{4 g} \frac{d g}{d r}\right) U^{s, p}(x, y)\right)=\left(s+\frac{r}{4 g} \frac{d g}{d r}\right) B^{p}\left(U^{s, p}(x, y)\right) \\
&+\sum_{1 \leq k \leq l_{3}} \sum_{\substack{\sigma(1)<\cdots<\sigma(k) \\
\sigma(k+1)<\cdots<\sigma\left(l_{3}\right)}}\left(\nabla_{j_{\sigma(1)}} \circ \cdots \circ \nabla_{j_{\sigma(k)}}\left(s+\frac{r}{4 g} \frac{d g}{d r}\right)\right) D^{p} B_{1} \circ \ldots \\
& \circ D^{p} B_{l_{1}+l_{2}} \circ \nabla_{j_{\sigma(k+1)}} \circ \ldots \circ \nabla_{j_{\sigma\left(l_{3}\right)}}\left(U^{s, p}(x, y)\right) .
\end{aligned}
$$

By the induction hypothesis we therefore get 
$\sum_{p=0}^{d}(-1)^{p} \operatorname{Tr}\left[B^{p}\left(\left(s+\frac{r}{4 g} \frac{d g}{d r}\right) U^{s, p}\right)\right](x, x)=s \sum_{p=0}^{d}(-1)^{p} \operatorname{Tr}\left[B^{p}\left(U^{s, p}\right)\right](x, x)$.

Similarly,

$$
\begin{aligned}
& \sum_{p=0}^{d}(-1)^{p} \operatorname{Tr}\left[B^{p} \circ g^{k j} \nabla_{j} \circ \nabla_{k}\left(U^{s-1, p}\right)\right](x, x)=0, \\
& \sum_{p=0}^{d}(-1)^{p} \operatorname{Tr}\left[B^{p} \circ g^{k j} \Gamma_{k j}^{a} \nabla_{a}\left(U^{s-1, p}\right)\right](x, x)=0 .
\end{aligned}
$$

Finally Lemma 3.3 together with the induction hypothesis gives

$$
\sum_{p=0}^{d}(-1)^{p} \operatorname{Tr}\left[B^{p} \circ D^{p} R\left(U^{s-1, p}\right)\right](x, x)=0
$$

By first applying the operator $B^{p}$ to both sides of equation (5.1) and then taking trace we thus obtain

$$
\left(l_{3}+s\right) \sum_{p=0}^{d}(-1)^{p} \operatorname{Tr}\left[B^{p}\left(U^{s, p}(x, y)\right)\right](x, x)=0 .
$$

Since $l_{3}+s>0$, we have $\sum_{p=0}^{d}(-1)^{p} \operatorname{Tr}\left[B^{p}\left(U^{s, p}(x, y)\right)\right](x, x)=0$, which completes the proof of Lemma 5.2.

Proof of Lemma 5.1. Using the Main Lemma 5.2, we shall now prove Lemma 5.1. As an immediate consequence of the main lemma we have

$$
\sum_{p=0}^{d}(-1)^{p} \operatorname{Tr} U^{i, p}(x, x)=0 \quad \text { for } i<n
$$

On the other hand, by equation (5.1) we obtain

$$
\begin{aligned}
n \sum_{p=0}^{d}( & -1)^{p} \operatorname{Tr} U^{n, p}(x, x) \\
= & \sum_{k, j} g^{k j}(x) \sum_{p=0}^{d}(-1)^{p} \operatorname{Tr}\left[\nabla_{k} \circ \nabla_{j}\left(U^{n-1, p}\right)\right](x, x) \\
& \quad-\sum_{k, j, a} g^{k j}(x) \Gamma_{k j}^{a}(x) \sum_{p=0}^{d}(-1)^{p} \operatorname{Tr}\left[\nabla_{a}\left(U^{n-1, p}\right)\right](x, x) \\
& +\sum_{p=0}^{d}(-1)^{p} \operatorname{Tr}\left[D^{p} R\left(U^{n-1, p}\right)\right](x, x)
\end{aligned}
$$

The first two terms on the right hand side of (5.3) are zero by Lemma 5.2 so that 


$$
\begin{aligned}
\sum_{p=0}^{d}(-1)^{p} \operatorname{Tr} U^{n, p}(x, x)=\frac{1}{n} \sum_{p=0}^{d}(-1)^{p} \operatorname{Tr}\left[D^{p} R\left(U^{n-1, p}\right)\right](x, x) \\
=\frac{1}{n(n-1)} \sum_{k, j} g^{k, j}(x) \sum_{p=0}^{d}(-1)^{p} \operatorname{Tr}\left[D^{p} R \circ \nabla_{j} \circ \nabla_{k}\left(U^{n-2, p}\right)\right](x, x) \\
\quad-\frac{1}{n(n-1)} \sum_{k, j, a} g^{k j}(x) \Gamma_{k j}^{a}(x) \sum_{p=0}^{d}(-1)^{p} \operatorname{Tr}\left[\nabla_{a} U^{n-2, p}\right](x, x) \\
\quad+\frac{1}{n(n-1)} \sum_{p=0}^{d}(-1)^{p} \operatorname{Tr}\left[D^{p} R \circ D^{p} R\left(U^{n-2, p}\right)\right](x, x), \quad \text { by (4) . }
\end{aligned}
$$

Again by Lemma 5.2 the first two terms on the right hand side of (5.4) are zero so that

$$
\sum_{p=0}^{d}(-1)^{p} \operatorname{Tr} U^{n, p}(x, x)=\frac{1}{n(n-1)} \sum_{p=0}^{d}(-1)^{p} \operatorname{Tr}\left[D^{p} R \circ D^{p} R\left(U^{n-2, p}\right)\right](x, x) .
$$

Proceeding thus we finally get

$$
\begin{aligned}
\sum_{p=0}^{d}(-1)^{p} \operatorname{Tr} U^{n, p}(x, x) & =\frac{1}{n !} \sum_{p=0}^{d}(-1)^{p}\left[\left(D^{p} R\right)^{n}\left(U^{0, p}\right)\right](x, x) \\
& =\frac{1}{n !} \sum_{p=0}^{d}(-1)^{p} \text { trace of the operator }\left(D^{p} R\right)_{x}^{n}
\end{aligned}
$$

Therefore by Lemma 2.3 we obtain

$$
\begin{aligned}
& \frac{1}{(4 \pi)^{n}} \sum_{p=0}^{d}(-1)^{p} \operatorname{Tr} U^{n, p}(x, x) \\
& =\frac{1}{2^{2 n} \pi^{n} n !} \sum_{\sigma, \delta} \varepsilon_{\sigma} \varepsilon_{\delta} R_{\delta(1) \sigma(1) \sigma(2) \delta(2)} \cdots R_{\delta(2 n-1) \sigma(2 n-1) \sigma(2 n) \delta(2 n)} \\
& =\frac{1}{2^{3 n} \pi^{n} n !} \sum_{\sigma, \delta} \varepsilon_{\sigma} \varepsilon_{\delta}\left(R_{\delta(1) \sigma(1) \sigma(2) \delta(2)}-R_{\delta(1) \sigma(2) \sigma(1) \delta(2)}\right) \cdots \\
& \quad \cdot\left(R_{\delta(2 n-1) \sigma(2 n-1) \sigma(2 n) \delta(2 n)}-R_{\delta(2 n-1) \sigma(2 n) \sigma(2 n-1) \delta(2 n)}\right) \\
& =\frac{(-1)^{n}}{2^{3 n} \pi^{n} n !} \sum_{\sigma, \delta} \varepsilon_{\sigma} \varepsilon_{\delta} R_{\delta(1) \delta(2) \sigma(1) \sigma(2)} \cdots R_{\delta(2 n-1) \delta(2 n) \sigma(2 n-1) \sigma(2 n)} \\
& \quad \text { by using the formula } R_{i j k l}-R_{i k j l}+R_{i l j k}=0 \\
& =C .
\end{aligned}
$$




\section{References}

[1] S. S. Chern, A simple intrinsic proof of the Gauss-Bonnet formula for closed Riemannian manifolds, Ann. of Math. 45 (1944) 747-752.

[2 ] H. P. McKean, Jr. \& I. M. Singer, Curvature and the eigenvalues of the Laplacian, J. Differential Geometry 1 (1967) 43-69.

[3] S. Minakshisundaram \& $\AA$. Pleijel, Some properties of the eigenfunctions of the Laplace operator on Riemannian manifolds, Canad. J. Math. 1 (1949) 242-256.

[ 4 ] E. Nelson, Tensor analysis, Princeton University Press, Princeton, 1967, 101-102.

[ 5 ] G. de Rham, Variétés différentiables, Hermann, Paris, 1955, 127-131.

Tata Institute, Bombay 
